2000-07

Isolation and characterisation of a cAMP-dependent protein kinase catalytic subunit gene from Plasmodium falciparum.

$\mathrm{Li}, \mathrm{J}$

http://hdl.handle.net/10026.1/10324

10.1016/s0166-6851(00)00242-5

Mol Biochem Parasitol

All content in PEARL is protected by copyright law. Author manuscripts are made available in accordance with publisher policies. Please cite only the published version using the details provided on the item record or document. In the absence of an open licence (e.g. Creative Commons), permissions for further reuse of content should be sought from the publisher or author. 


\title{
Isolation and characterisation of a cAMP-dependent protein kinase catalytic subunit gene from Plasmodium falciparum ${ }^{\text {is }}$
}

\author{
Ji-Liang Li *, Lynne S. Cox \\ Department of Biochemistry, University of Oxford, South Parks Road, Oxford OX1 3QU, UK \\ Received 3 February 2000; received in revised form 27 March 2000; accepted 6 April 2000
}

Keywords: Plasmodium falciparum; Malaria; cAMP-dependent protein kinase; Chromosome

The cAMP-dependent protein kinase (PKA) is a key element of the signal transduction pathway by means of the second messenger cAMP. cAMP, generated as a result of activation of membranebound adenylyl cyclases by $G$ protein-coupled surface receptors, exerts nearly all of its effects by activation of PKA [1]. In most organisms, PKA is a heterotetramer consisting of two catalytic and two regulatory subunits [1]. In Dictyostelium, however, PKA is a heterodimer composed of a

Abbreviations: PKA, cAMP-dependent protein kinase; PfPKAc, Plasmodium falciparum cAMP-dependent protein kinase catalytic subunit; PKIs, the heat-stable protein kinase inhibitors; PfPP- $\alpha$, Plasmodium falciparum protein serine/ threonine phosphatase $\alpha$; PfPP- $\beta$, Plasmodium falciparum protein serine/threonine phosphatase $\beta$; PFGE, pulse-field gel electrophoresis.

Note: Nucleotide sequence data reported in this paper are available in the GenBank ${ }^{\mathrm{TM}}$, EMBL and DDJB databases under the accession number AF126719.

* Corresponding author. Present address: Institute of Molecular Medicine, University of Oxford, John Radcliffe Hospital, Oxford OX3 9DS, UK. Tel.: + 44-1865-222419; fax: + 44-1865-222431.

E-mail address: lij@icrf.icnet.uk (J.-L. Li). catalytic and a regulatory subunit [2]. Kinase activity of the catalytic subunit is inhibited by the regulatory subunit. Binding of cAMP to the regulatory subunit alters its affinity for the catalytic subunit, and under physiological conditions, the catalytic subunit dissociates from the regulatory subunit and subsequently phosphorylates many substrate proteins. In mammalian cells, there are three isoforms of the catalytic subunits $(\mathrm{C} \alpha, \mathrm{C} \beta$ and $\mathrm{C} \gamma$ ) and four isoforms of the regulatory subunits (RI $\alpha$, RI $\beta$, RII $\alpha$ and RII $\beta$ ) [3] and the tissue-specific expression and assembly of these kinase isoforms are postulated to result in the diverse cellular responses to cAMP [1]. Apart from the regulatory subunits, the heat-stable protein kinase inhibitors (PKIs) are also able to bind the catalytic subunit with a high affinity and subsequently inhibit the kinase activity [1].

Plasmodium falciparum has a complex life cycle involving two different hosts and interactions with multiple cell types. The molecular and cellular mechanisms involved in regulation of proliferation and development of $P$. falciparum are un- 
clear. However, inhibitors of protein kinases and phosphatases can interfere with parasite growth [4], suggesting a requirement for phosphorylation-dephosphorylation in control of the parasite life cycle. Reversible phosphorylation has been shown to play an important role in invasion of erythrocytes by merozoites [5] and during intraerythrocytic growth and development of the parasite [6]. The cAMP- and $\mathrm{Ca}^{2+}$-dependent protein kinase activities of $P$. falciparum have been detected in cytosolic extracts of the asexual stages of $P$. falciparum [7]. We are interested in signal transduction pathways involved in the cell growth and differentiation of $P$. falciparum. One of our immediate goals has been to isolate the various protein kinases from the parasite. Recently, we have reported several stage-specific genes encoding protein serine/threonine kinases $[8,9]$. In this paper, we describe the identification and characterisation of a novel gene encoding the $P$. falciparum PKA catalytic subunit (PfPKAc). PfPKAc is expressed specifically in the asexual stage, indicating that it may be important in regulating the processes of asexual stage growth and differentiation.

In the $P$. falciparum tag database, there is an expressed sequence tag $(0648 \mathrm{c} 3)$ that encodes a protein fragment with a high sequence homology to the catalytic subunits of the PKA family. To isolate the whole gene encoding PfPKAc, two specific primers, CK1 (5'-ATGGATCATTCAAAGATGACTCTTAC-3', 1664-1689) (to obtain further sequence in the $3^{\prime}$ direction) and $\mathrm{CK} 2$ (5'-GTAGCGAGAATAACTCTACCAAAGG-

$3^{\prime}, 1261-1285$ ) (to obtain further sequence in the $5^{\prime}$ direction), were constructed on the basis of the tag sequence and used in PCR to screen Vectorette libraries [8] (Fig. 1(A)). Three fragments (CK1-BclI, CK2-TaqI and CK2-AluI) were obtained and sequenced. The CK2-TaqI fragment, as predicted, covered the whole CK2-AluI sequence and contained a putative ATG start codon, while the CK1-BclI lacked a putative stop codon but permitted construction of the CK3 primer (5'-TTGAACGTCGGACATGGAAAAGCC-3', 2112-2135) that gave rise to the CK3-DraI fragment. The known sequence data made it possible to construct the CK5 primer
(5'-TTTGATGAAGAAATTGTTGTCTCACG-

$3^{\prime}, 2603-2628$ ) for further sreening of vectorette libraries. Based on the sequence of CK5-Sau3AI, the CK7 primer (5'-GGTACAAGAAGATTTAACTATAGCTG-3', 2789-2814) was synthesised and then employed to produce the CK7-TaqI fragment. Sequence analysis revealed a putative TAG stop codon in CK7-TaqI. In order to confirm the sequence obtained from the overlapping fragments, a pair of primers, CKB (5'AGGATCCCATATGCAGTTTATTAAAAATTT GCAGC-3', 946-971) and CKE (5-TAAGCTTACCAATCATAAAATGGATCATTTTC-3',

2829-2854), which covered the putative start and stop codons respectively, was used to amplify the full-length gene from genomic DNA and the PCR product was sequenced in both strands (Fig. 1(A)). The sequence derived from overlapping PCR fragments consists of $3706 \mathrm{bp}$ and contains five exons, four introns and $5^{\prime}$ and $3^{\prime}$ untranslated regions. The proposed coding region of the Pfp$k a c$ gene starts with an ATG codon at nucleotide 947 and terminates with a TAG codon at nucleotide 2853. In both flanking untranslated regions, there are in-frame stop codons for all three possible reading frames. The sequence and codon usage in the coding region are typical for a $P$. falciparum gene. The $\mathrm{A}+\mathrm{T}$ contents of both flanking (946 bp at $5^{\prime}$ and $854 \mathrm{bp}$ at $3^{\prime}$ ) and four putative intron non-coding regions are characteristically higher than those of the exon coding regions. Five continued putative polyadenylation signals (AATAAA) are found at nucleotide +67 , downstream of the TAG termination codon. A diffuse $\mathrm{G}+\mathrm{T}$-rich sequence is also found at nucleotide +82 , downstream of the fifth polyadenylation signal. The four proposed introns, ranging from 137 to 321 nucleotides in length, interrupt the coding region. The highly conserved dinucleotides GT and AG, found at eukaryotic intron boundaries [10], define these intervening sequences. Long runs of poly(AT), poly(T) and poly(A) are present in the introns. To verify the size and location of these presumed introns, PCR was performed against cDNA using CKB and CKE primers (data not shown). Sequencing of the RT-PCR products confirmed the precise exon-intron boundaries. 
(A)

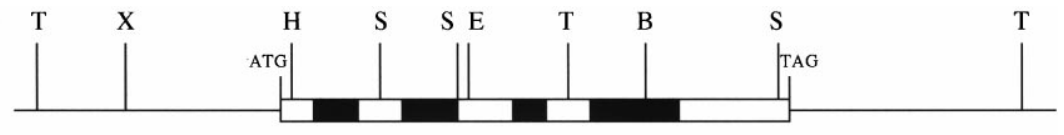

Tag $(0648 \mathrm{c} 3)$

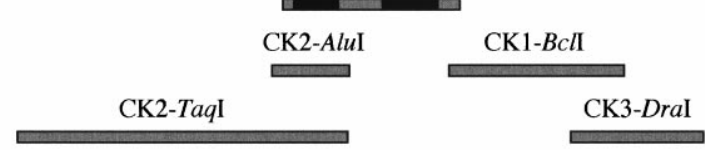

CK5-Sau3AI

CK7-TaqI

CKB-CKE

CKB-CKE

(B)

\begin{tabular}{|c|c|c|}
\hline PfPKAC & 1 & $-----M Q F I K N L Q L N K K K D S D S S E Q V L T N K K N K M K Y E$ \\
\hline Ac & 1 & ---MIQFLKNLQLYKKR--ETSDIKPNTKKSKMKYE \\
\hline \multirow[t]{2}{*}{ tPKAC $-\alpha$} & 1 & NAAAAKKGSEQESVKEFLAKAKE--DFLKKWENPAQNTAHLI \\
\hline & & $::{ }^{\star}::$ \\
\hline & 50 & LATYKNGNYPPVAIKRFEKCKI IRQKQVDHVFSERKILNYINI \\
\hline & 49 & LATYKNEDLPPVAIKRFEKSKI IKQKQVDHVFSERKI LNYINH \\
\hline \multirow[t]{3}{*}{ tPKAC $-\alpha$} & 59 & LVKHMETGN-HYAMKI LDKQKVVKLKQIEHTLNEKRILQAVNE \\
\hline & & $\star^{\star}:^{\star}::^{\star}{ }^{\star}:::^{\star \star}::^{\star} \quad:^{\star}::^{\star \star}::^{\star}$ \\
\hline & & I I \\
\hline & 110 & LVLEFVIGGEFFTFLRRNKRFPNDVGCFYAAQIVLIFEYLQS \\
\hline & 109 & LVLEFVIGGEFFTFLRRNKRFPNDVGCFYAAQIVLI FEYLQSI \\
\hline \multirow[t]{3}{*}{ tPKAC- $\alpha$} & 118 & MVMEYVPGGEMFSHLRRIGRFSEPHARFYAAQIVLTFEYLHS \\
\hline & & 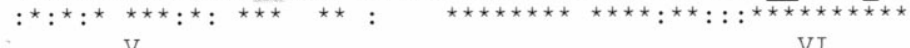 \\
\hline & & V \\
\hline & 170 & GFIKMTDEGFAKIVETRTYTLCGTPEYIAPEILLNVGHGKA \\
\hline & 169 & FIKMTDFGFAKVVNTRTYTLCGTPEY IAPEILLNAGHGKA \\
\hline \multirow[t]{2}{*}{ tPKAC- $\alpha$} & 178 & GYIQVTDFGFAKRVKGRTWTLCGTPEYLA \\
\hline & & 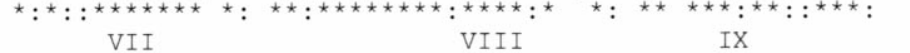 \\
\hline & 230 & FYANEPLLIYQKILEGI IYFPKFLDNNCKHLMKKLLSHDLTKRYGNLKKGAQN \\
\hline Ac & 229 & FYANEPLLIYQKILEGI IYFPKFLDNNCKHLMKKLLSHDLTKRYGN \\
\hline \multirow[t]{2}{*}{ tPKAC $-\alpha$} & 238 & FFADQPIQIYEKIVSGKVRFPSHFSSDLKDLLRNLLQVDLTKRFGNLKNGVND \\
\hline & & 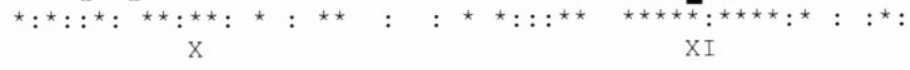 \\
\hline & 290 & YKPKYKNIFDSS \\
\hline C & 28 & PYKPKYKNI FDASNFEKVQED---LS \\
\hline \multirow[t]{2}{*}{$-\mathrm{PKAC}-\alpha$} & 298 & ATTDWIAIYQRKVEAPFI PKFKGPGDTSNFDDYEEEE IRVS INEKCGKEFSEF \\
\hline & & $: \quad:^{\star} \quad::::^{\star}:{ }^{\star}:{ }^{\star \star}:{ }^{\star} \quad{ }^{\star}:{ }^{\star \star \star}: \quad:^{\star}: \quad::^{\star} \quad:^{\star} \quad:$ \\
\hline
\end{tabular}

Fig. 1. (A) A schematic representation of a partial restriction map of the Pfpkac gene and the overlapping fragments used to determine its nucleotide sequence. B, BclI; E, EcoRI; H, HincII; S, Sau3AI; T, TaqI; X, XbaI. The open boxes indicate the exon coding regions of the Pfpkac gene and the black boxes represent the introns. The fragments of CK1-BclI, CK2-AluI, CK2-TaqI, CK3-DraI, CK5-Sau3AI and CK7-TaqI are derived from genomic DNA. The CKB-CKE fragment is amplified from genomic DNA and cDNA, respectively. (B) Alignment of the predicted amino acid sequence of PfPKAc with those of PyPKAc and BtPKAc- $\alpha$. The GenBank ${ }^{\mathrm{TM}} / \mathrm{EMBL} / \mathrm{DDJB}$ database accession numbers are as follows: P. falciparum PfPKAc, AF126719; P. yoelii PyPKAc, D45849; and Bos taurus (bovine) BtPKAc- $\alpha$, X67154. Sequences were aligned with the CLUSTAL W (1.60) multiple sequence alignment programme. The amino acid residues are numbered to the left of the sequence. Identical residues are indicated with asterisks and conservative changes indicated with dots. The eleven canonical subdomains of protein kinases [12] are indicated by roman numerals. The residues conserved in the catalytic domain of the protein kinase family are highlighted with solid black and the residues involved in interaction with the regulatory subunits or PKIs are shaded with grey. 
The open reading frame resulting from removal of the four introns encodes a protein of 342 amino acids (Fig. 1(B)) with a predicted molecular mass of approximately $40.2 \mathrm{kDa}$ and an isoelectric point 9.02. Database searches revealed that the amino acid sequence of PfPKAc shares 68 94\% similarity and 46-88\% identity with kinases in the PKA family with the highest homology (88\% identity, 94\% similarity) to the $P$. yoelii PKA catalytic subunit (PyPKAc) [11]. It is noteworthy that the gene encoding PyPKAc also contains four introns [11]. Although the size and composition of the introns are different between the two genes, all four introns are located in the same places corresponding to the deduced proteins.

PfPKAc contains all 11 conserved subdomains of the protein kinase family [12] (see Fig. 1(B)) and has almost all of the characteristic features of a kinase [12-14]. The sequences (RDLKPEN) in subdomain VI and (GTPEYIAPE) in subdomain VIII indicate that the predicted protein is a serine/ threonine kinase rather than a tyrosine kinase [12]. Important residues of the catalytic subunits required for interaction with the regulatory subunits and PKIs and for phosphorylation regulation have been identified by genetic screens, biochemical mutagenesis and crystalisation studies both in mammalian cells and in yeast [15-20]. PfPKAc has most of these essential amino acid residues. However, close inspection of the sequence revealed that PfPKAc is different to its homologues in mammalian cells in several respects. First, PfPKAc does not contain the FXXF motif that has been found in nearly all PKAs [21]. The two phenylalanine residues in this motif have been known to participate in the anchoring to the core of the C-terminal end of the mouse catalytic subunit [13] and the mutation of one or both of the residues in the Dictyostelium catalyitc subunit results in a strong decrease of catalytic activity and stability of the protein [21]. In addition, the highly conserved residues W196 and K213 (corresponding to the residue numbers for the bovine PKAc- $\alpha$ ), which have been demonstrated to be important for interaction with the regulatiory subunits in yeast and mammalian cells $[17,19]$, are replaced by Y188 and V205 in PfPKAc, respec- tively, suggesting that the potential regulatory subunits of PfPKAc might differ to some extent from those in mammalian cells. Indeed, two cAMP-binding proteins with the molecular mass of $53 \mathrm{kDa}$ and $49 \mathrm{kDa}$ respectively have been detected in $P$. falciparum by both photoaffinity labeling with $\left[{ }^{32} \mathrm{P}\right] 8-\mathrm{N}_{3}$-cAMP and affinity chromatography of metabolically $\left[{ }^{35}\right.$ S $]$ methionine-labeled cytosol of cAMP-agarose [7,22] although the molecular bases of these two proteins have not been verified. Moreover, PfPKAc lacks the sequence of DDYEEEE near the C-terminus, which is thought to be necessary for the binding with PKIs, implying that the potential PKIs of PfPKAc would be different from those in human cells. Therefore, the PfPKA holoenzyme could represent a promising target for development of new anti-malarial drugs.

To determine the copy number of the Pfpkac gene in the $P$. falciparum genome, 3D7A genomic DNA was digested with a number of restriction enzymes and analysed by Southern blotting. Hybridisation of the CKB-CKE (see Fig. 1(A)) DNA probe revealed a single band in digests with $A c c \mathrm{I}, B a m \mathrm{HI}$ or $E c o \mathrm{RV}$, for which there is no any restriction site in the $\mathrm{CKB}-\mathrm{CKE}$ fragment, and two bands in digests with $B c l$ I or Eco RI, for which only one restriction site exists in CKB-CKE (Fig. 2(A)). These results suggest strongly that PfPKAc is encoded by a single copy gene in the parasite genome. To investigate the chromosome location of the Pfpkac gene, P. falciparum (3D7A and T996) chromosomes were resolved on the CHEF gel system, blotted onto a nylon membrane and hybridised with the CKB-CKE DNA probe. A single band was detected corresponding to chromosome 9 (Fig. 2(B)). This was confirmed by probing the same blot with a control probe derived from the $P f p p-\beta$ gene (Fig. 2(B)), which is known to be located on chromosome 9 [23].

To obtain some information on how Pfpkac mRNA levels are regulated during parasite development and differentiation, a northern blot containing equal quantities of total RNA prepared from cultures enriched in stage III to stage $\mathrm{V}$ gametocytes and from mixed asexual erythrocytic stages was probed with the CKB-CKE cDNA fragment (see Fig. 1(A)). A single transcript of 

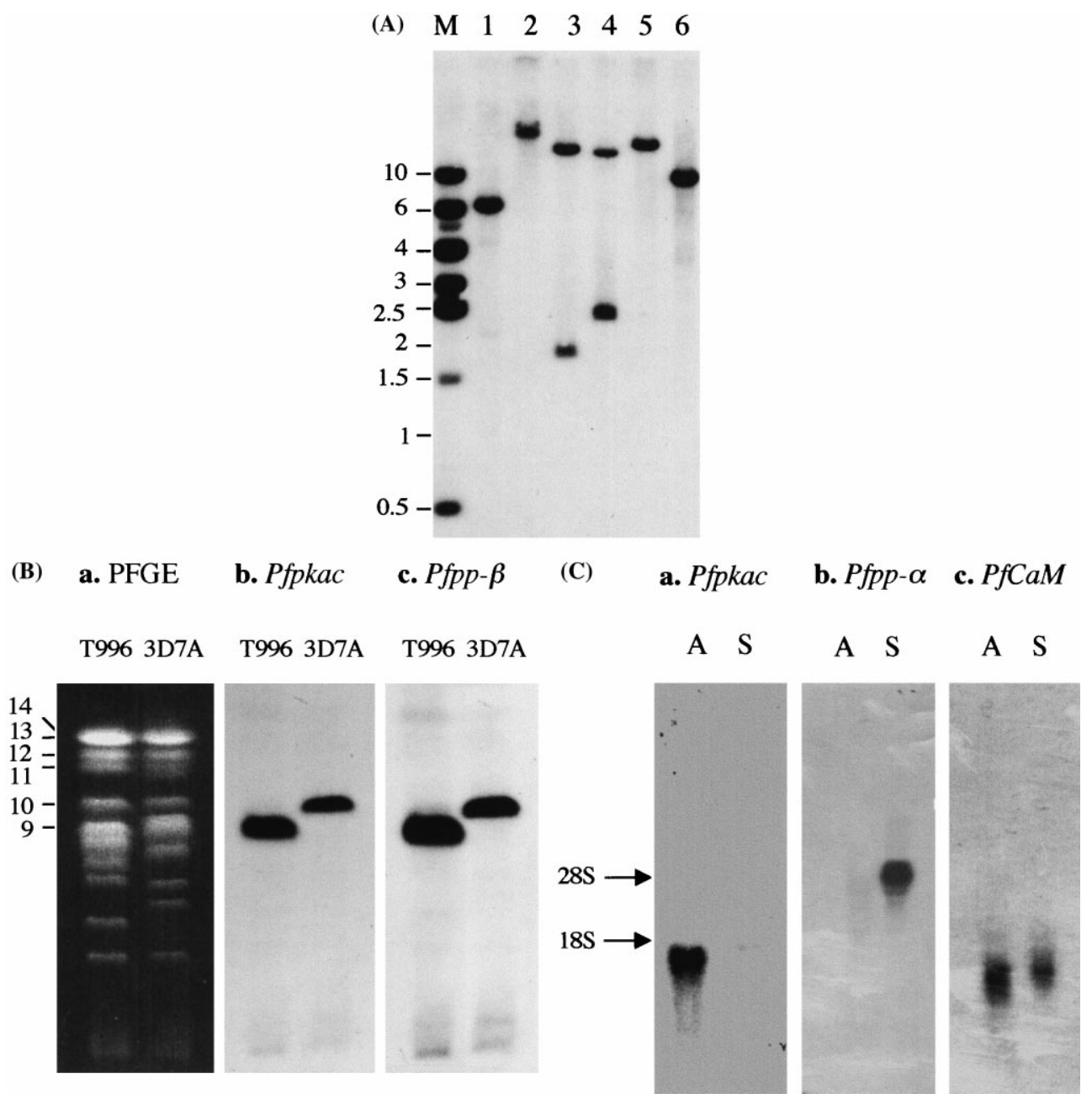

Fig. 2. (A) Southern blot analysis of the Pfpkac gene. $4 \mu \mathrm{g}$ of genomic DNA from P. falciparum clone 3D7A were digested with restriction enzymes, electrophoresed on a $1.0 \%$ agarose gel, transferred onto a nylon membrane, and probed with the CKB-CKE DNA fragment of Pfpkac. Lanes 1-6 correspond to digests with $A c c$ I, Bam HI, BclI, Eco RI, Eco RV and Hinc II. The sizes of 1-Kb DNA markers (M) are given in kilobase pairs to the left. (B) Chromosomal localisation of the Pfpkac gene. Parasite chromosomes from P. falciparum T996 and 3D7A were separated by pulse-field gel electrophoresis, stained with ethidium bromide, blotted onto a nylon membrane and hybridised with radiolabeled probes. According to the yeast chromosome markers and hybridisation of several $P$. falciparum chromosome marker genes, the positions of chromosome 9-14 were identified and indicated on the ethidium bromide-stained gel (a). The Pfpkac gene (b) and the Pfpp- $\beta$ gene (c) hybridized to chromosome 9. (C) Northern blot analysis of the $P$ fpkac gene. $10 \mu \mathrm{g}$ of total RNA extracted from asexual erythrocytic stages (A) and sexual erythrocytic stage (S) of $P$. falciparum (3D7A) were fractionated in a denaturing formaldehyde gel, blotted onto a nylon membrane and hybridised to radiolabeled probes. The positions of $P$. falciparum rRNA subunits (18S and 28S) are indicated by arrows. (a, b and c) are autoradiographs of the membrane probed with the Pfpkac gene (the CKB-CKE cDNA fragment), the Pfpp- $\alpha$ gene [28] and the calmodulin gene (PfCaM), and exposed for 1, 24 and 96 h, respectively. Pfpkac detected a transcript of approximately 1800 nucleotides in asexual stage, $P f p p-\alpha$ hybridised with a band of approximately 3900 nucleotides in sexual stage and PfCaM hybridised with two bands of 1300 and 1000 nucleotides from the parasite. 
approximately 1800 nucleotides in size was detected only in the lane containing the asexual stage RNA, migrating ahead of the $18 \mathrm{~S}$ ribosomal RNA band (Fig. 2(C)). The result suggests that PfPKAc is involved in asexual stage-specific events. It has been demonstrated that addition of $1 \mathrm{mM}$ cAMP inhibited maturation of ring stages while trophozoites and schizonts remained relatively unaffected [24]. cAMP was shown to enhance the differentiation of $P$. falciparum to gametocytes by addition of cAMP or dibutyryl cAMP (a membrane-permeable cAMP analogue) to stationary phase cultures of the parasite [25]. Treatment of $P$. falciparum cultures with cAMP phosphodiesterase inhibitors, caffeine [26] and 8bromo-cAMP [27], increased gametocyte production. Interestingly, PKA activity has been known to be higher in gametocyte-producing clones than in gametocyte-non-producing clones [22]. Taken together, it is concluded that PfPKAc plays an important role in control of the parasite maturation and differentiation. The recombinant PfPKAc produced in E. coli (data not shown) will facilitate the identification of the upstream regulators and downstream substrates of PfPKAc and consequently afford new insight on the regulatory mechanisms of the parasite growth and differentiation by the cAMP signal transduction pathway.

\section{Acknowledgements}

We are very grateful to Dr David A. Baker for his PFGE blot. This work was supported in part by the Royal Society and the Cancer Research Campaign.

\section{References}

[1] Taylor SS, Beichler JA, Yonemoto W. cAMP-dependent protein kinase: framework for a diverse family of regulatory enzymes. Annu Rev Biochem 1990;59:971-1005.

[2] Loomis WF. Role of PKA in the timing of developmental events in Dictyostelium cells. Microbiol Mol Biol Rev 1998;62:684-94.

[3] Doskeland SO, Maronde E, Gjersten B. The genetic subtypes of cAMP-dependent protien kinase - functionally different or redundant? Biophim Biophys Acta 1993;1178:249-58.
[4] Ward GE, Fujioka H, Aikawa M, Miller LH. Staurosporine inhibits invasion of erythrocytes by malarial merozoites. Exp Parasitol 1994;79:480-7.

[5] Rangachari K, Dluzewski A, Wilson RJM, Gratzer WB. Control of malarial invasion by phosphorylation of the host cell membrane cytoskeleton. Nature 1986;324:364-5.

[6] Yuthavong Y, Limpaiboon T. The relationship of phosphorylation of membrane proteins with the osmotic fragility and filterability of Plasmodium berghei-infected mouse erythrocytes. Biochim Biophys Acta 1987;929:278-87.

[7] Read LK, Mikkelsen RB. Cyclic AMP- and $\mathrm{Ca}^{2+}$-dependent protein kinases in Plasmodium falciparum. Exp Parasitol 1990;71:39-48.

[8] Li JL, Robson KJH, Chen JL, Targett GAT, Baker DA. Pfmrk, a MO15-related protein kinase from Plasmodium falciparum: gene cloning, sequence, stage-specific expression and chromosome localization. Eur $\mathbf{J}$ Biochem 1996;241:805-13.

[9] Li JL, Baker DA, Cox LS. Sexual stage-specific expression of a third calcium-dependent protein kinase from Plasmodium falciparum. Biochim Biophys Acta 2000;1491:341-9.

[10] Padgett RA, Grabowski PJ, Konarska MM, Seiler S, Sharp PA. Splicing of messenger RNA precursors. Ann Rev Biochem 1986;55:1119-50.

[11] Saito-Ito A, He S, Kimura K, Matsumura T, Tanabe K. Cloning and structural analysis of the gene for cAMP-dependent protien kinase catalytic subunit from Plasmodium yoelii. Biochim Biophys Acta 1995;1269:1-5.

[12] Hanks SK, Quinn AM, Hunter T. The protein kinase family: conserved features and deduced phylogeny of the catalytic domains. Science 1988;241:42-52.

[13] Knighton DR, Zheng J, TenEyck LF, Ashford FA, Xuong NH, Taylor SS, Sowadski JM. Crystal structure of the catalytic subunit of cyclic adenosine monophosphatedependent protein kinase. Science 1991;253:407-14.

[14] Zhang F, Strand A, Robbins D, Cobb MH, Goldsmith EJ. Atomic structure of the MAP kinase ERK2 at $2.3 \AA$ resolution. Nature 1994;367:704-11.

[15] Knighton DR, Zheng J, TenEyck LF, Xuong N, Taylor SS, Sowadski JM. Structure of a peptide inhibitor bound to the catalytic subunit of cyclic adenosine monophosphate-dependent protein kinase. Science 1991;253:414-20.

[16] Gibbs CS, Knighton DR, Sowadski JM, Taylor S, Zoller MJ. Systematic mutational analysis of cAMP-dependent protein kinase identifies unregulated catalytic subunits and defines regions important for the recognition of the regulatory subunit. J Biol Chem 1992;267:4806-14.

[17] Orellana SA, McKnight GS. Mutations in the catalytic subunit of cAMP-dependent protein kinase result in unregulated biological activity. Proc Natl Acad Sci USA 1992;89:4726-30.

[18] Wen W, Taylor SS. High affinity binding of the heat-stable protein kinase inhibitor to the catalytic subunit of cAMP-dependent protein kinase is selectively abolished by mutation of Arg133. J Biol Chem 1994;269:8423-30. 
[19] Gibson RG, Ji-Buechler Y, Taylor SS. Identification of electrostatic interaction sites between the regulatory and catalytic subunits of cyclic AMP-dependent protein kinase. Protein Sci 1997;6:1825-34.

[20] Cauthron RD, Carter KB, Liauw S, Steinberg RA. Physiological phosphorylation of protein kinase A at Thr-197 is by a protein kinase A kinase. Mol Cell Biol 1998;18:1416-23.

[21] Etchebehere LC, Van Bemmelen MXP, Anjard D, Traincard F, Assemat K, Reymond C, Veron M. The catalytic subunit of Dictyostelium cAMP-dependent protein kinase: role of the $\mathrm{N}$-terminal domain and of the C-terminal residues in catalytic activity and stability. Eur J Biochem 1997;248:820-6.

[22] Read LK, Mikkelsen RB. Comparison of adenylate cyclase and cAMP-dependent protein kinase in gametocytogenic and nongametocytogenic clones of Plasmodium falciparum. J Parasitol 1991;77:346-52.

[23] Li JL, Baker DA. Protein phosphatase $\beta$, a putative type-2A protein phosphatase from the human malaria parasite Plasmodium falciparum. Eur J Biochem 1997;249:98-106.

[24] Inselburg J. Stage-specific inhibitory effect of cyclic AMP on asexual maturation and gametocyte fromation of Plasmodium falciparum. J Parasitol 1983;69:592-7.

[25] Kaushal DC, Carter R, Miller LH, Krishna G. Gametocytogenesis by malaria parasites in continuous culture. Nature 1980;286:490-2.

[26] Brockelman CR. Conditions favoring gametocytogenesis in the continuous culture of Plasmodium falciparum. J Protozool 1982;29:454-8.

[27] Trager W, Gill GS. Plasmodium falciparum gametocyte formation in vitro: its stimulation by phorbol diesters and by 8-bromo cyclic adenosine monophosphate. J Protozool 1989;36:451-4.

[28] Li JL, Baker DA. A putative protein serine/threonine phosphatase from Plasmodium falciparum contains a large $\mathrm{N}$-terminal extension and five unique inserts in the catalytic domain. Mol Biochem Parasitol 1998;95:287-95. 